\title{
Long-range properties and data validity for hydrogeological time series: the case of the Paglia river
}

\author{
Marcel Ausloos* \\ University of Leicester - School of Management. \\ Email: marcel.ausloos@ulg.ac.be \\ Roy Cerqueti \\ University of Macerata - Department of Economics and Law. \\ Email: roy.cerqueti@unimc.it \\ Claudio Lupi \\ University of Molise - Department of Economics. \\ Email: lupi@unimol.it
}

October 24, 2016

\begin{abstract}
This paper explores a large collection of about 377,000 observations, spanning more than 20 years with a frequency of 30 minutes, of the streamflow of the Paglia river, in central Italy. We analyze the longterm persistence properties of the series by computing the Hurst exponent, not only in its original form but also under an evolutionary point of view by analyzing the Hurst exponents over a rolling windows basis. The methodological tool adopted for the persistence is the detrended fluctuation analysis (DFA), which is classically known as suitable for our purpose. As an ancillary exploration, we implement a control on the data validity by assessing if the data exhibit the regularity stated by Benford's law. Results are interesting under different viewpoints. First, we show that the Paglia river streamflow exhibits periodicities which broadly suggest the existence of some common behaviour with El Niño and the North Atlantic Oscillations: this specifically points to a (not necessarily direct) effect of these oceanic phenomena on the hydrogeological equilibria of very far geographical zones: however, such an hypothesis needs further analyses to be validated. Second, the series of streamflows shows an antipersistent behaviour. Third, data are not consistent with Benford's law: this suggests that the measurement criteria should be opportunely revised. Fourth, the streamflow
\end{abstract}

${ }^{*}$ Corresponding author 
distribution is well approximated by a discrete generalized Beta distribution: this is well in accordance with the measured streamflows being the outcome of a complex system.

Keywords: River streamflow, Hurst exponent, Benford's law, Detrended fluctuation analysis, Discrete generalized Beta distribution.

\section{Introduction}

Hydrogeological paths exhibit often a complex behaviour. Such irregularities are driven by the presence of several factors of uncertainty in the climate dynamics. River streamflows represent paradigmatic examples of this evidence. In fact, human activities and natural events drive the fluctuations, sometimes with catastrophic effects, of such phenomena.

This paper elaborates some crucial observations from these premises. In particular, we develop an analysis of the streamflow of the Paglia river, a major tributary of Tiber, whose watercourse is entirely localized in Central Italy. Data are the official measurements of the streamflow of the river at Ponte dell'Adunata, near Orvieto, a historical town in Umbria region. The time interval covered by the measurements is January 1st, 1992 (12:00am) - May 13, 2014 (h11:30pm), and the periodicity of the observations is 30 minutes. However, the pervasive presence of missing values in the first part of the series prevented us from using the data prior to November 2, 1992 (h12:30pm).

We specifically focus on the so-called long run dependence property of the series, which gives a thoughtful view of the behaviour of the autocorrelation function. Such a statistical property can be suitably employed for making forecasts on the future evolution of the streamflow. The perspective presented here is in line with the original, path-breaking study by ?, who explored the long-run dependence of the runoffs of the Nile river. The analysis is based on the assessment of the value of the constant $H \in(0,1)$ - the so-called Hurst exponent - which represents the rate of decay of the autocorrelation function of the series as a function of the time-lag. If $H>1 / 2$, the series is said to present a persistent behaviour, which basically means that the history of the past will "statistically" repeat in the future; $H<1 / 2$ stands for antipersistence, the opposite of persistence; $H=1 / 2$ is the pure random case.

Since its inception, several contributions dealing with the assessment of the Hurst exponent in a number of very different contexts have appeared in the literature; a complete list is too long to be mentioned here.

In detecting long-run dependence, river discharges are of particular interest. In fact, they offer very specific time series with peculiar features. First of all, they have periodicities, with different periods. Such a property 
is due to the strong relationship between the river streamflow dynamics, the weather and the precipitation, but also to the effects of the global warming over the hydrogeological systems of the planet Earth (see, e.g., ???????).

Under the point of view of the human activities, it is self-evident how the fluctuations of the streamflow of any river affect (positively or negatively) the socio-economic system of the surrounding areas. In this respect, the Paglia river and the municipality of Orvieto represent very interesting cases to treat. In fact, there is a high level of hydrogeological risk related to Paglia's floods in the Orvieto area: the last important flood took place on November 12, 2012, causing injuries and huge damages to the local economy. An effective plan to deal with this recurring phenomenon should necessarily move from a deep knowledge of the dynamics of the river and a forecast of when and how a flood will take place in the future.

From a purely technical viewpoint, we proceed by applying the Detrended Fluctuation Analysis (DFA) - introduced by ? - which is classically acknowledged to be a powerful tool for the estimation of the Hurst exponent, whence long term persistence effects, mainly in the presence of nonstationarities. The appeal of the DFA lies in its conceptualization. Indeed, the underlying theoretical framework can be found in the theory of random walks (see ??). In such a context, time series are opportunely aggregated. This reduces the noise level due to biases in measurements.

In the context of DFA applications, it is worth mentioning ????.

As an ancillary analysis, we also consider the "validity" of the data measured at Ponte dell'Adunata. We do not infirm its interest, of course, but we wish to point some possible defects impairing a finer analysis as that presented here. In fact, the subsequent discussion and conclusions might be the first of this sort in the present context. For this purpose, and in accordance with ? observations of river streamflows in the USA, we check if data fit Benford's law (?).

In fact, such an empirical rule (on the logarithmic distribution of the first digit in lengthy data) has been found to hold in a wide spectrum of cases (see, e.g., ????????, and the references therein). When violated, the presence of some sort of data manipulation or mistakes in data collection has to be debated.

The rest of the paper is organized as follows: in the next Section (Sect. 2) we introduce the dataset and the methodologies. In Section 3 we present and discuss the results. In the last Section (Sect. 4), we offer some concluding remarks.

\section{Data and methodologies}

The Paglia river is an important right-side tributary of Tiber, the third longest river in Italy. It is about $86 \mathrm{~km}$-long, with many tributaries along 


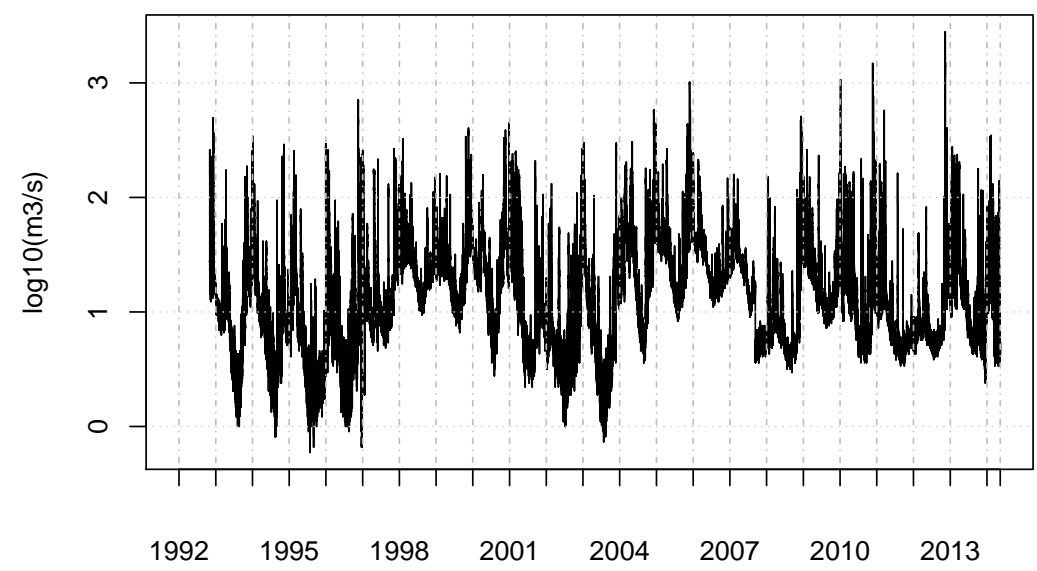

Figure 1: Streamflow (expressed in $\log _{10}\left(\mathrm{~m}^{3} / \mathrm{s}\right)$ ) of the Paglia river measured at every half hour between November 2, 1992 and May 13, 2014. Ticks on the $\mathrm{x}$-axis identify the beginning of the years, except the the last tick that indicates the last available observation.

its course. It is characterized by a very variable streamflow (see Table 1 and Figure 1) which gives Paglia a crucial role in determining the floods of Tiber. Data, obtained from an hydrometer station run by the Umbria regional authority, measure the streamflow (expressed in $\mathrm{m}^{3} / \mathrm{s}$ ) of the Paglia river at Ponte dell'Adunata, just downstream of the confluence of Chiani river, a 42km-long left-side tributary of Paglia.

Because of the presence of many long measurement interruptions during the first ten months of 1992, in our analysis we consider measurements recorded at each half hour starting on November 2, 1992 (h12:30pm), until May 13, 2014 (h11:30pm). There are still a few missing values in the selected period (in 7 occurrences the interruption of the measurements lasted for more than 2 days). Such missing values have been linearly interpolated on the series expressed in logarithms. ${ }^{1}$ The resulting series (plotted in logs in Figure 1) includes 377,399 observations.

Data have been analyzed under several perspectives: first, we carry out a graphical analysis of the main features of the streamflows.

Then, we estimate the spectrum of the daily averages, in order to assess the presence of seasonal and non-seasonal periodicities.

For what concerns the estimation of the Hurst exponent, we perform a detrended fluctuation analysis (DFA) over 90-day long rolling windows. At

\footnotetext{
${ }^{1}$ All computations have been carried out using $\mathrm{R}$ ver. $3.3 .1(?)$ and packages benford.analysis (?), fractal (?), ggplot2 (?), imputeTS (?), moments (?), psd (?), xts (?).
} 


\begin{tabular}{cccccc}
\hline \hline Min. & 1st Q. & Median & Mean & 3rd Q. & Max. \\
0.59 & 6.29 & 12.31 & 20.53 & 23.93 & 2794.00 \\
\hline$\sigma$ & Skewness & Kurtosis & Date of Max. & Date of Min. & Data points \\
34.63 & 20.81 & 989.48 & $2012 / 11 / 12$ & $1995 / 07 / 28$ & 377399 \\
\hline \hline
\end{tabular}

Table 1: Main descriptive statistics of the Paglia river streamflow $\left(\mathrm{m}^{3} / \mathrm{s}\right)$ as measured at Ponte dell'Adunata, near Orvieto (November 2, 1992 - May $13,2014)$.

each iteration the window is moved forward by a 1-day step. For a more intuitive visualization, when needed, windows will be numbered according to a chronological criterion. Specifically, we start from window 1 - the one at the beginning of the sample period. Then, we shift the window by one day and add 1 to the windows counter at any shift. According to this mechanism, window 1 is the one spanning days $1-90$, window 2 spans days 2-91, window 3 insists on days 3-92, and so on. The Hurst exponent, $H$, is estimated in each window, resulting in a series of 7,773 estimated values of the Hurst exponent. In order to check the sensitivity of the results on the window length, we repeat the same analysis using 180 and 400-day long windows.

We also carry out a check for "data validity". For this purpose, we adopt the point of view of ?, who suggest that streamflow statistics of US rivers are broadly consistent with Benford's law. For this reason we check if the data gathered for the Paglia river are also consistent with Benford's law. This part of the analysis is carried out on the observed data only, without any imputation of missing values. In particular we carry out a first-two digits test, which is more informative than the combination of both the first digit and the second digit tests (see e.g. ?, especially Chapter 4).

Finally, we check if the observed data approximately follow a discrete generalized Beta distribution (DGBD), typical of the output of complex systems (?).

\section{Results and discussion}

The streamflow series expressed in logarithms shows several features of the Paglia river fluctuations (see Figure 1). However, more information can be gathered by looking at the basic monthly statistics of the data: it is evident that the series exhibits some periodicity, visually two regimes: a stationary one, representing the "normal" streamflow of the Paglia river, and a peaked one, which captures the cases of floods. This is nicely exemplified by displaying the monthly distribution of the water flow as provided in Figure 2: significant variations are apparent. Indeed, the streamflow of the river is more powerful in winter than in summer, and this is in line with the 


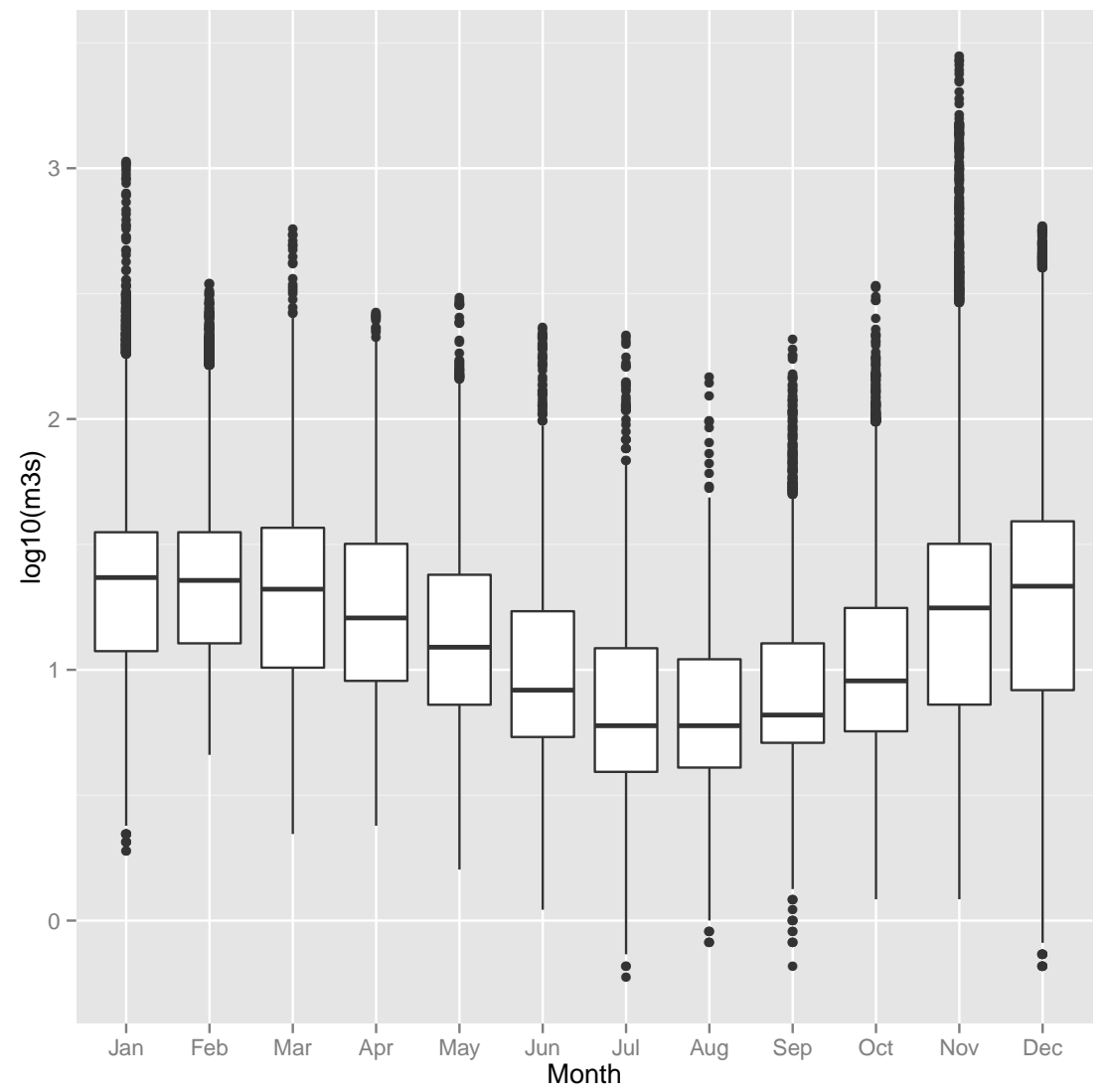

Figure 2: Distribution of the streamflow $\left(\log _{10}\left(\mathrm{~m}^{3} / \mathrm{s}\right)\right)$ of the Paglia river by month. 


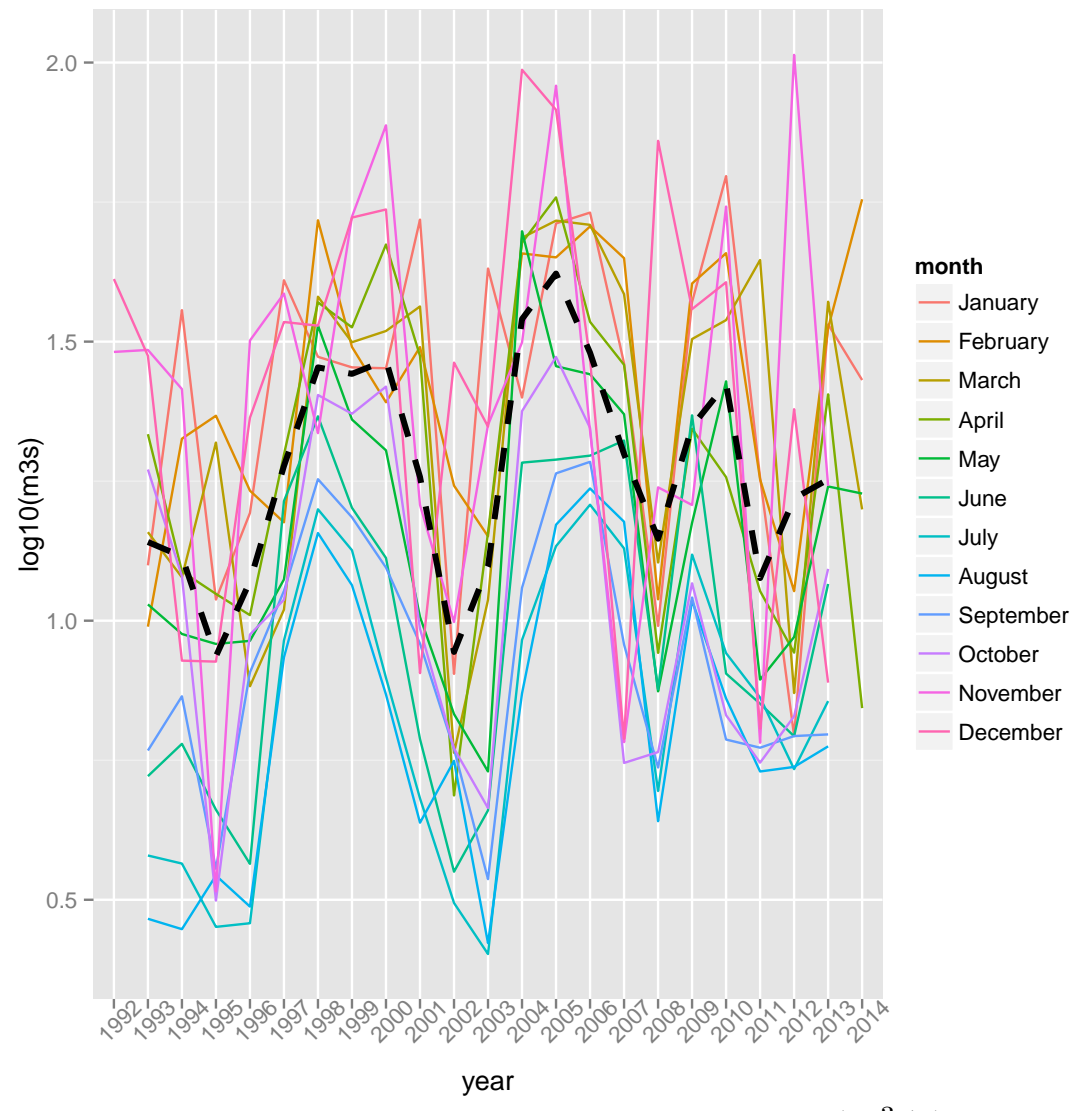

Figure 3: Evolution of monthly average streamflow $\left(\mathrm{m}^{3} / \mathrm{s}\right)$ of the Paglia river. The black dashed line represents the yearly average.

standard precipitation cycle in Italy, which exhibits a periodicity associated to the seasons. However, the highest streamflow peaks are all concentrated in November, even if the median value of the streamflow in that month is smaller than in the other winter months. This observation suggests that the maximum intensity of precipitations in the area is historically located in November, despite this month not being the most rainy one.

The evolution of the monthly averages is plotted in Figure 3. If the median streamflow is considered instead of the average, the overall picture remains substantially unchanged and is possibly even clearer (Figure 4). A cyclical pattern is apparent from the data, with a cycle whose period is about $5-7$ years. This result suggests the existence of a relationship between rivers streamflow fluctuations and the periodicity of the oscillations of $\mathrm{El}$ Niño, which is recurrent on irregular intervals with an average period of 3-4 years (see, e.g., ??). However, it is worth to point out that the observed periodicities of these different phenomena do not perfectly match, indicating that further investigations are needed for a confirmation of this hypothesis. 


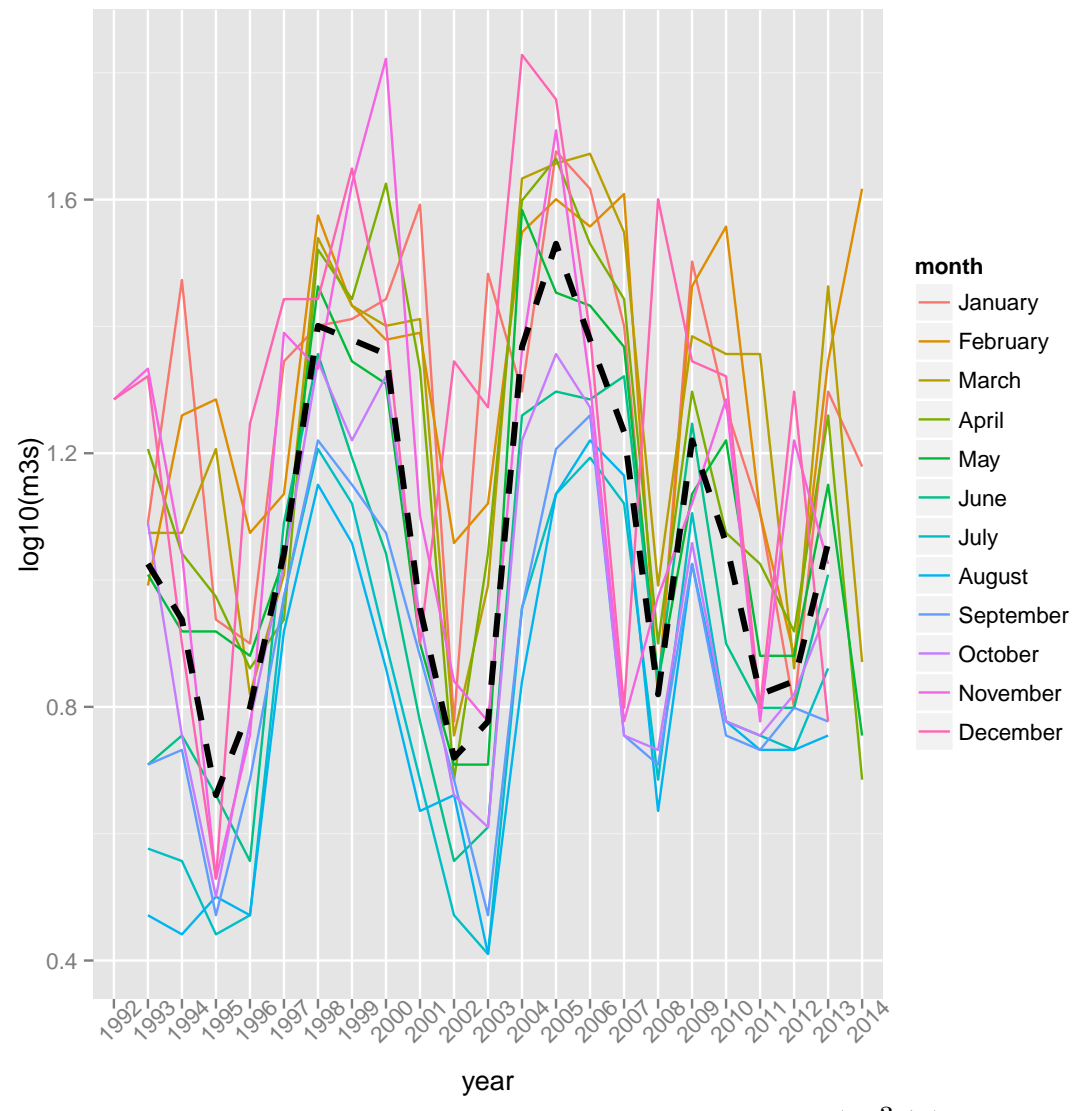

Figure 4: Evolution of monthly median streamflow $\left(\mathrm{m}^{3} / \mathrm{s}\right)$ of the Paglia river. The black dashed line represents the yearly median.

Furthermore, such a periodicity is also broadly in accordance with that of the North Atlantic Oscillations, whose fluctuations show a fairly regular periodic behaviour over a time interval of $6-8$ years (see, e.g., ?).

Equally interesting is the evolution of the monthly maxima, reported in Figure 5, where a positive trend seems to exist in the recent annual maxima of the streamflow. This means that the intensity of the streamflow maxima has grown with respect to time in the last few years. This finding is in line with the overall climate change, which represents a severe and debated concern.

The rolling estimates of the Hurst exponent are plotted in Figure 6: at first sight this figure seems to suggest the presence of frequent large oscillations of the estimated values of the Hurst exponent in adjacent windows. In fact, this is not the case and this impression is mainly due to the high density of data points along the x-axis in the graph. A closer examination of the estimated values of the Hurst exponent reveals that there are few relatively large oscillations, related to an extreme event entering (or leaving) 


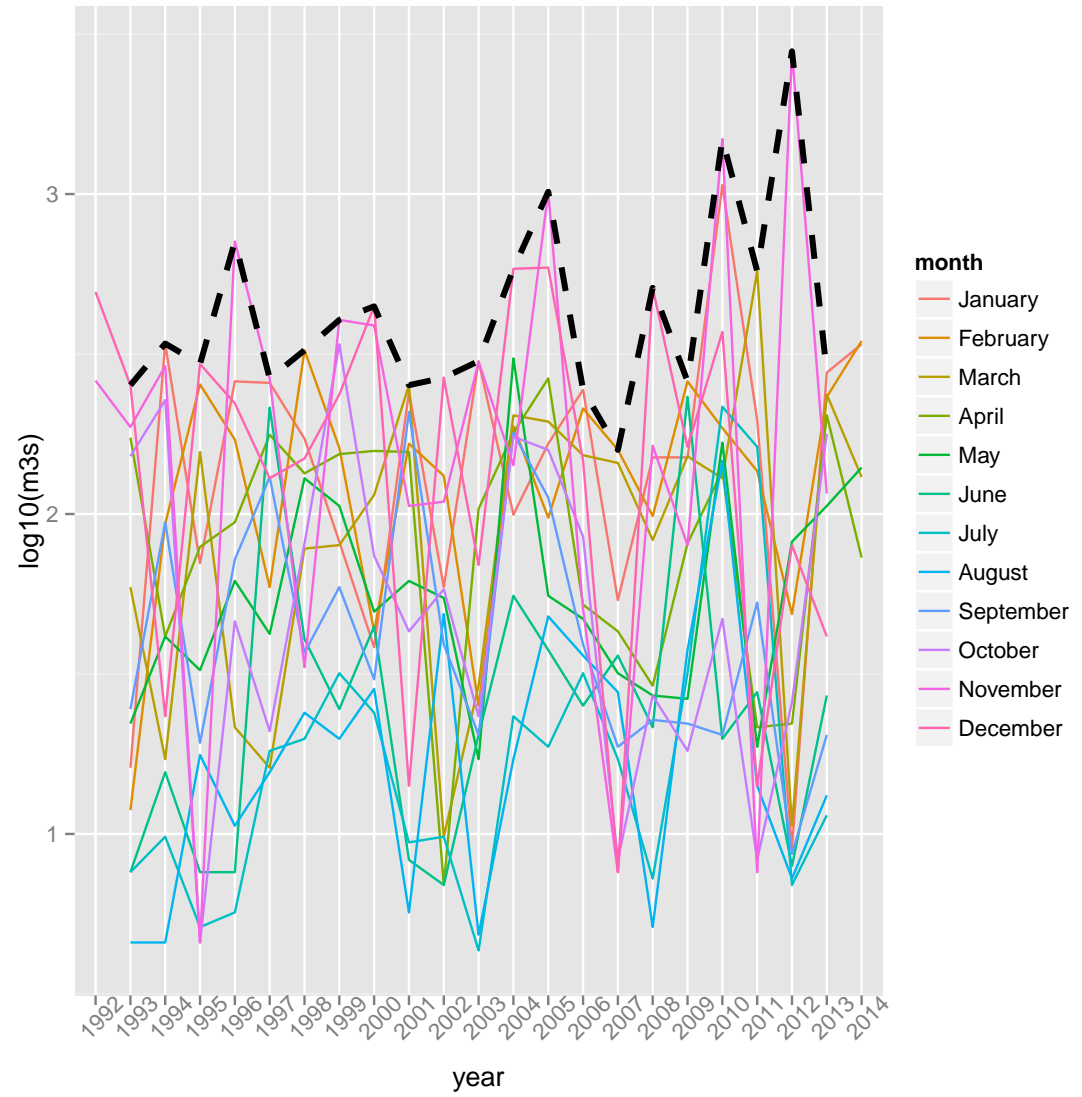

Figure 5: Evolution of monthly maxima $\left(\mathrm{m}^{3} / \mathrm{s}\right)$ of the Paglia river. The black dashed line represents the yearly maximum. 

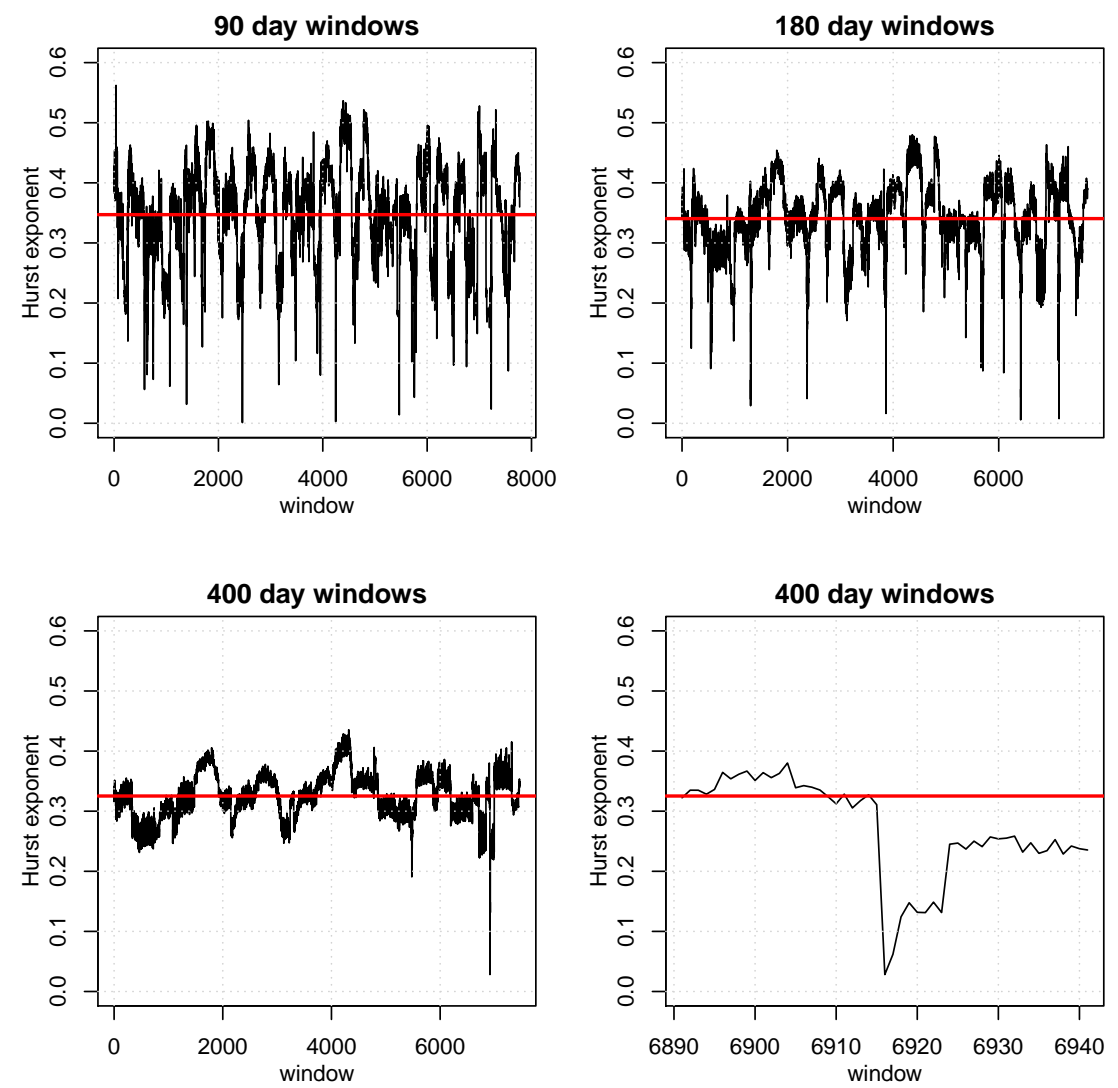

Figure 6: Estimated Hurst exponent over 90, 180, and 400-day long rolling windows. At each iteration the window is moved forward by a 1 day step. On the $\mathrm{x}$-axis is reported chronological order given to windows. The horizontal red line is the average estimate of the Hurst exponent over all the windows. The fourth (lower-right) panel focuses on a subset of windows centred on the negative peak of the 400-day long windows estimates, corresponding to the first window containing November 12, 2012. 
the estimation window. This effect is clearly visible in the fourth panel of Figure 6 that focuses on a subset of windows in the neighbourhood of the negative spike depicted in the 400-day long windows panel. Here it can be seen that the value of the Hurst exponent is fairly stable until the data of the flood of November 12, 2012 enter the estimation window: in coincidence with the new data entering the window, the estimated value of the Hurst exponent drops.

When considering 90-day long windows, $90 \%$ of the variations of the estimates across adjacent windows belong to the interval $(-0.043,0.046)$ and about $3.6 \%$ of them are larger than 0.05 in absolute value, whereas only $0.4 \%$ are larger than 0.1 (less than one third of the average value). Furthermore, $90 \%$ of the differences across adjacent 180 -day long windows belong to the interval $(-0.032,0.034)$ and less than $0.1 \%$ of the variations are larger than 0.1 in absolute value: when 400-day windows are considered, these values become $(-0.023,0.022)$ and $0.03 \%$, respectively (see Table 2$)$. Note also that the estimated values are more stable for longer windows, as expected.

It is interesting also to observe that the average Hurst exponent over the rolling windows is about $1 / 3$ (see again Table 2 and Figure 6 ). This value means that the series of the Paglia river streamflows is highly antipersistent. By analogy with financial econometrics based on DFA and time evolution of the Hurst exponent, this observation should lead to constructive risk assessment measures, but an application or discussion of this forecasting process falls outside the framework of the present paper. Uncertainty associated with the estimates is small, as can be observed from Table 2 .

The estimates of the Hurst exponent seem to show a cyclical pattern, too. For this reason we carry out a spectral analysis of the series of the estimated Hurst exponents (see Figure 7) using an adaptive, sine multitaper power spectral density estimation method (?).

We are not now in the position to offer a definitive answer to this phenomenon. The apparent periodicity of the estimated Hurst exponent is probably influenced by the intrinsic features of the river streamflow: in particular, we observe that $2-3$ days is the time interval required for the streamflow to return to the "normal" level preceding a peak, in the absence of further atmospheric phenomena. This is well illustrated in Figure 8, where the dynamics of the streamflow in the neighbourhood of a number of peaks is compared: for ease of comparison, the peak heights are normalized to have the same range. We conjecture that the nonlinear, fairly recurrent, dynamics highlighted in Figure 8 may lay behind some of the observed periodicities of the Hurst exponent. This is not the only possible explanation, of course, and we acknowledge that the influence of several factors affecting the estimation of the Hurst exponent though DFA may be large and such to determine possible periodicities in the rolling estimates. To the best of our knowledge, a list of such factors should necessarily include the size of 


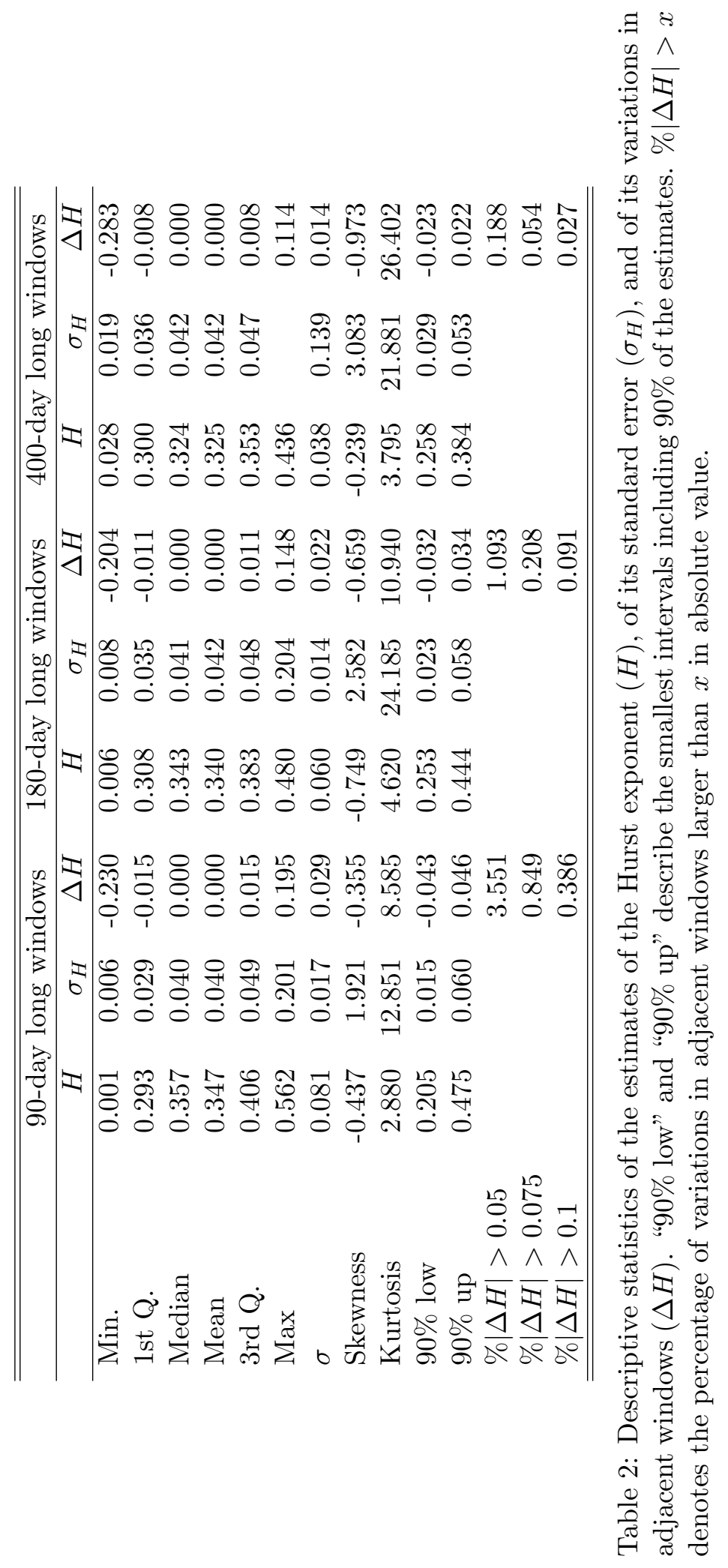



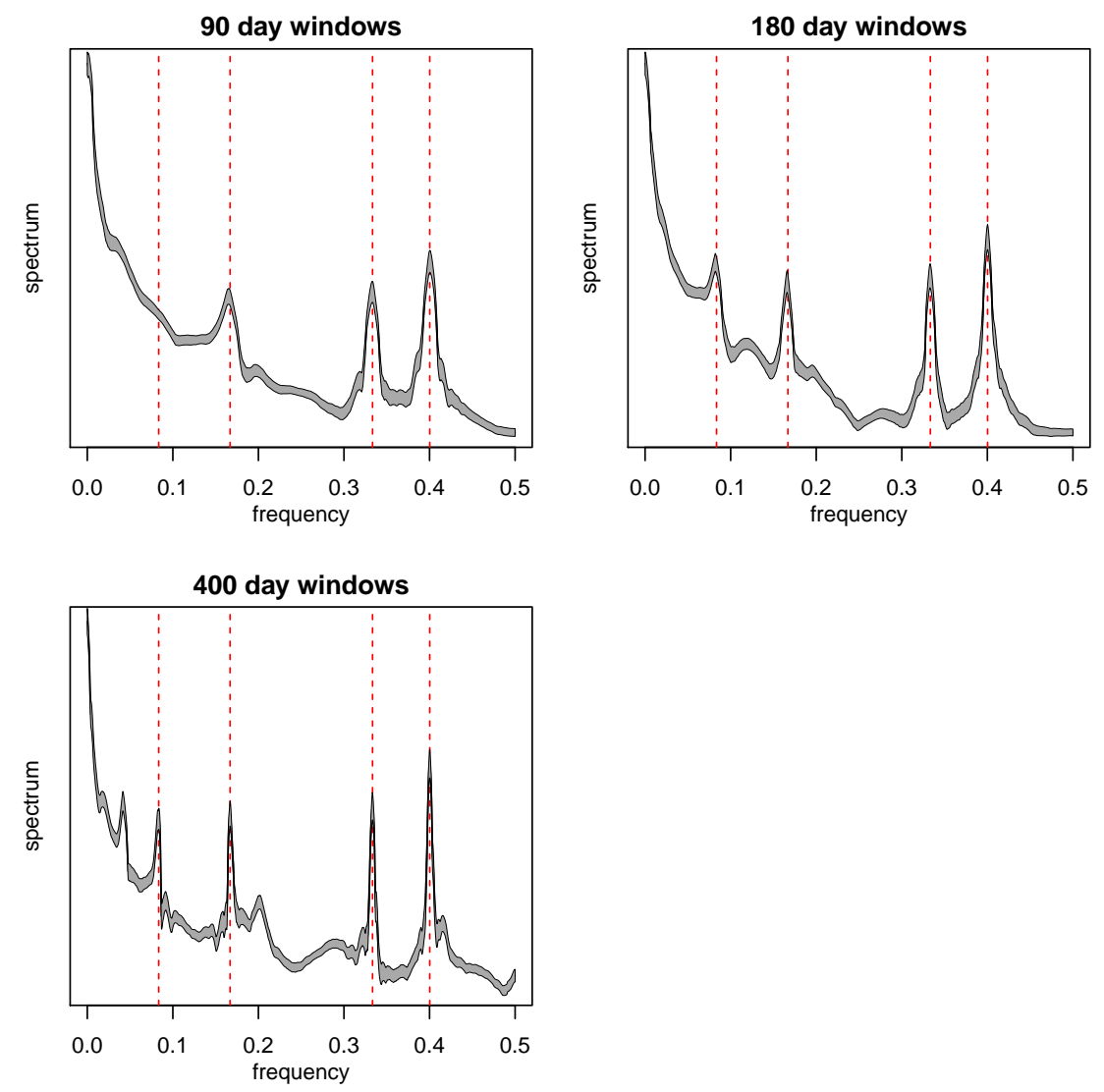

Figure 7: Estimated spectra of the series of the estimated Hurst exponents. The grey areas represent spectral uncertainties for $95 \%$ coverage probability. Peaks (from left to right) correspond to periodicities of about 12, 6, 3, and 2.5 days, respectively. 


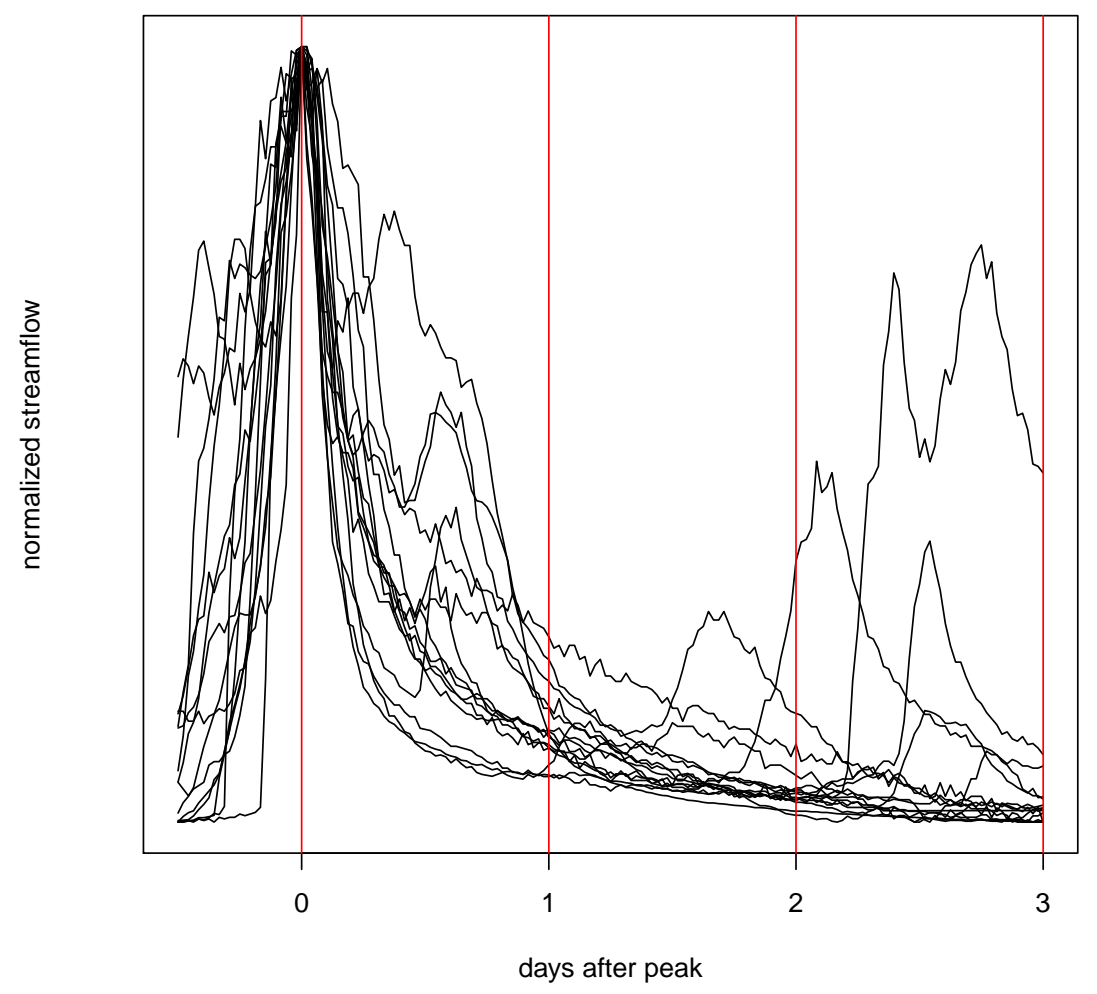

Figure 8: Comparison of the streamflow dynamics in the neighbourhood of a number of peaks. The x-axis represent the distance from the peak expressed in days. The $\mathrm{y}$-axis is the streamflow, normalized to have the same range across peaks.

the time window, the number of data points in the window, the position of the windows on the time interval, the presence of heteroskedasticity in the original data. The literature lists important contributions dealing with estimation problems of the Hurst exponent and proposing methods beyond the DFA (see, e.g., ??) or with different tools like moving average techniques (see, e.g., ??). Although this is beyond the scope of the present paper, the observed behaviour of the spectrum of the Hurst exponent suggests to carry out further investigations on the estimation procedure of the Hurst exponent, possibly along the lines advocated in the contributions mentioned above. At any rate, the order of magnitude of the Hurst exponent given by the DFA is incontestable.

We want now to focus on a potential weakness in the data recording process. The validity check of the data, implemented through the consistency with Benford's law, leads us to observe a substantial failure: data seem to vi- 

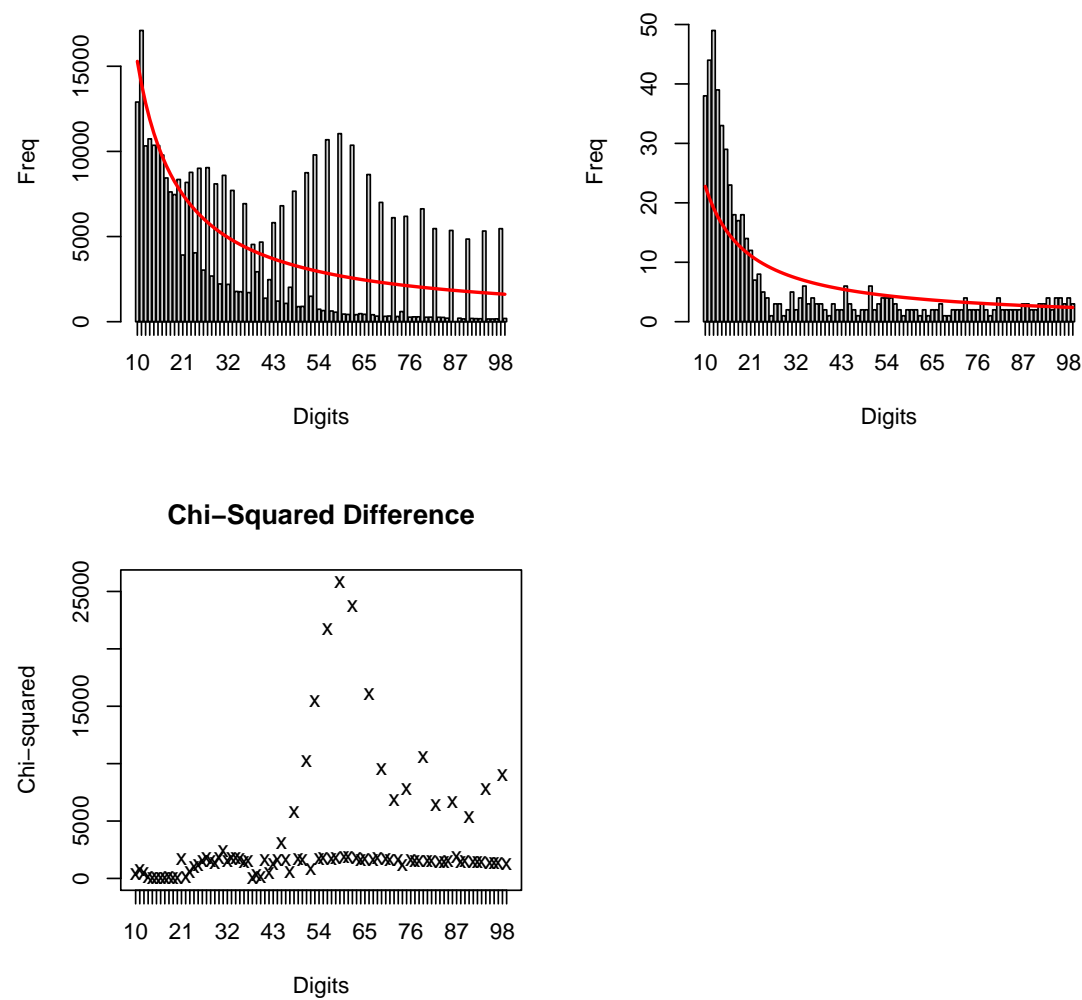

Figure 9: Benford's law first-two digits tests carried out on streamflow data $\left(\mathrm{m}^{3} / \mathrm{s}\right)$. Histograms represent the actual data distribution; the red solid curve is the theoretical Benford distribution.

olate Benford's law (see Figure 9). Recall that Benford's law (BL) describes the frequency distribution of leading digits in large data sets. In particular, Benford's law on the first digit (BL1) (??) states that the distribution of the first digit is more concentrated on smaller values: the digit "1" has the highest frequency, "9" the lowest frequency. The first digit distribution follows a logarithmic law:

$$
P(d)=\log _{10}\left(1+\frac{1}{d}\right), \quad d=1,2, \ldots, 9,
$$

where $P(d)$ is the probability that the first digit is equal to $d$ in the data set; $\log _{10}$ being the logarithm in base 10 .

There is a reasonable interpretation of the outcome of the inconsistency of the data with the regularity imposed by Benford's law. Flows $\left(\mathrm{m}^{3} / \mathrm{s}\right)$ are computed from water height, which is the really monitored quantity. However, water height is recorded with one centimetre precision, so that many repeated values are possible. In fact, the two most common values 

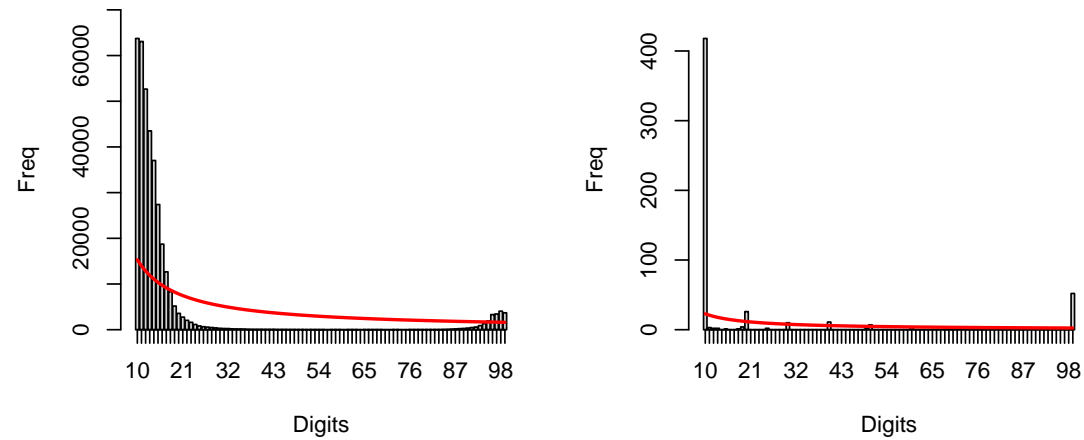

Chi-Squared Difference

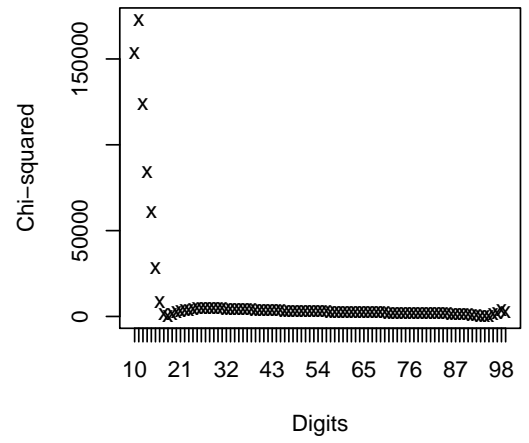

Figure 10: Benford's law first-two digits tests carried out on measured water heights $(\mathrm{cm})$. Histograms represent the actual data distribution; the red solid curve is the theoretical Benford distribution.

are repeated more than 10,000 times (see Table 3). The analysis repeated on water heights (measured in centimetres) reveals that the distribution of water heights does not conform to Benford's law either (see Figure 10). In fact, this is practically annoying when looking for streamflow fluctuations in predictive analyses.

Finally, looking for other important data regularities, we argue that a plot of the (log-)size against the rank of the river's streamflow (see Figure 11) suggests that this relation can be well approximated by a discrete generalized Beta distribution (DGBD) of the form

$$
f(r)=\frac{A(N+1-r)^{b}}{r^{a}}
$$

where $r$ is the $\operatorname{rank}, N:=\max (r)$, and $A, a$, and $b$ are parameters to be estimated from the data. ${ }^{2}$ This is indeed the case, and the $R^{2}$ of the

\footnotetext{
${ }^{2}$ Parameters can be estimated by Ordinary Least Squares from the model in logarithms.
} 


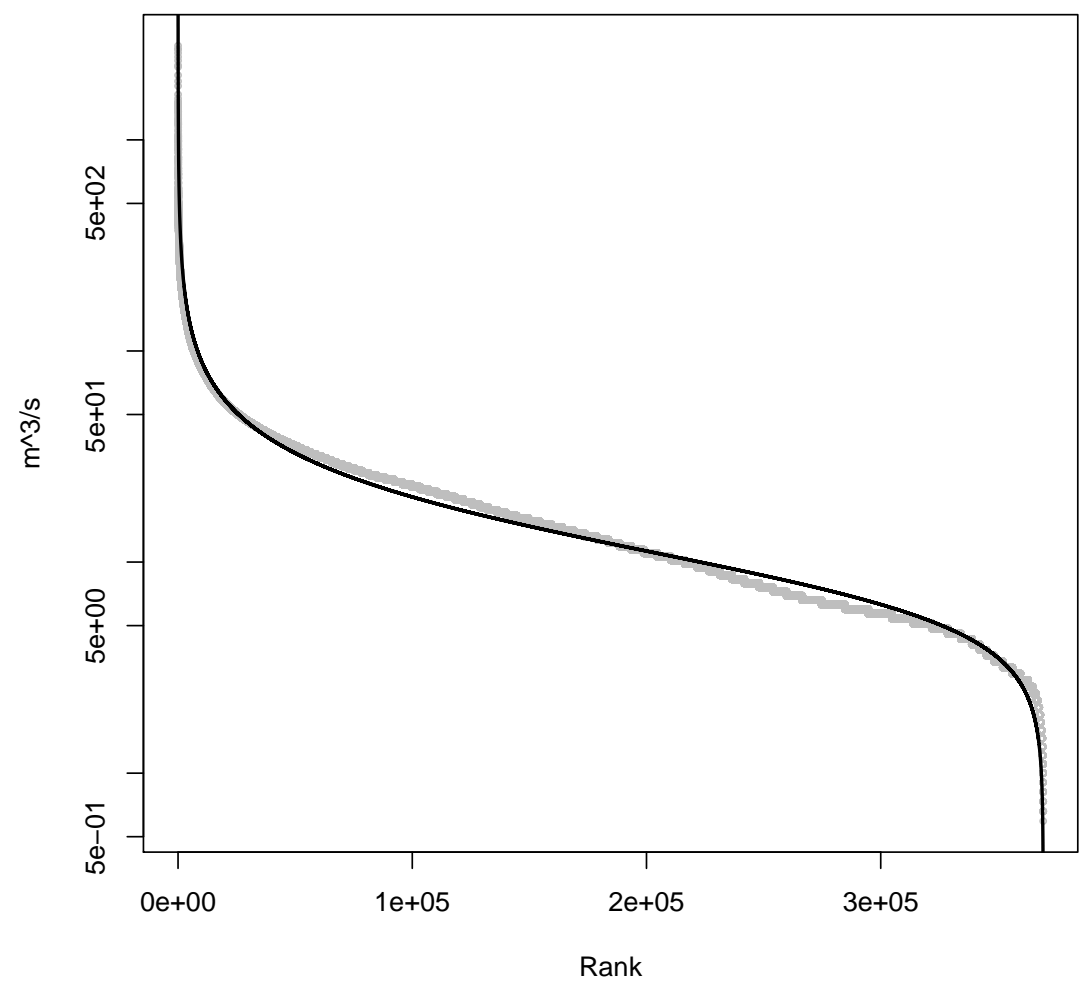

Figure 11: Rank-size relation of the Paglia river streamflow (data points are represented in grey). The continuous curve is the fit of a discrete generalized Beta distribution with $\left(A, a, b, R^{2}\right)=(163.117,0.596,0.382,0.986)$.

approximating function is $R^{2} \approx 0.986$, confirming the "universality" of the DGBD suggested in other fields of investigation (see, e.g., ?), particularly in relation to complex phenomena characterized by the co-existence of many subsystems whose interactions produce the observed outcome (?).

\section{Conclusions}

This paper presents the analysis of the Paglia river streamflow. The dataset used is of large size: the considered series has 30-minutes period over a timespan of more than 22 years. The data series is antipersistent, with an average Hurst exponent of about $1 / 3$. This gives a precise information on how one can do forecast on the overflow of the considered river. Furthermore, we show that in recent years there has been an increasing trend in the maxima of the river streamflows, and a periodicity which broadly suggests a connection of the streamflows with El Niño and the North Atlantic Oscillations. However, 


\begin{tabular}{rr}
\hline \hline $\mathrm{m}^{3} / \mathrm{s}$ & freq \\
\hline 5.9833 & 10061 \\
5.6859 & 10014 \\
6.2890 & 9940 \\
5.3968 & 9086 \\
6.6030 & 8263 \\
5.1161 & 7978 \\
4.8436 & 6784 \\
6.9253 & 6698 \\
7.9420 & 6361 \\
7.5948 & 5881 \\
\hline \hline
\end{tabular}

Table 3: Frequencies of the ten most common values.

more accurate measurements of the streamflow could be useful in having a more consistent dataset. We also show that the Paglia river streamflow is well represented by a discrete generalized beta distribution, congruent with the observed river discharge being the result of the interaction of many complex subsystems.

\section{Acknowledgements}

We would like to thank three anonymous referees for their constructive comments that helped us to substantially improve the paper upon the previous versions. We are pleased to thank Guido Calenda and Elena Volpi for having provided us with the data. Funding from the Italian Ministry of Education, University and Research (PRIN grant code 201002AXKAJ_005) is gratefully acknowledged. 